\title{
SÉANCE DU 11 JUIN 1909
}

Présidence de M. II. LECOMTE, Premier Vice-présinent.

M. F. Camus, vice-secrétaire, donne lecture du procèsverbal de la séance précédente. dont la rédaction est adoptée.

MI. Colte, Guillochon et Lavergne, récemment admis membres de la Société, ont envoyé des lettres de remerciement.

\section{DONS FAITS A LA SOCIÉTE}

Awano (S.), Veber die Benetzbarkieit der Blätter.

Bertrand (Paul), Étude sur la fronde des $Z_{y}$ goptéridées.

Bonati, Contribution à létude du genre Mazus.

- Sur quelques Pídiculaires chinoises nouvelles.

Brocq-Rousseu et Gain (Edm.), Sur la présence de l'amylase dans les vieilles graines.

- Oxydases et peroxydases des graines.

Comère (Paul), De la coloration anormale des Diatomées épiphytes.

Ceillier (Remi), Recherches sur l'influence de l'humidité du substratum sur les caractères du sclérenchyme de la racine des Fougères. - Résumé.

Constantin et abbé P. Gave, Flore populaire de la Savoie, $\mathbf{1}^{\text {re }}$ partie. Dictionnaire des noms populaires des plantes.

Coppey, Les Muscinées des environs de Nancy, $1^{\text {er fascicule. }}$

Dismier, Une Mousse nouvelle pour Maine-et-Loire, Fissidens Curnowii Mitt.

Ducomet (V.), Recherches sur quelques maladies des plantes cultivees.

Erwin Janchen, Die Cistaceen Oesterreich-Ungarns.

Farn (Edith N.). Contributions to a Catalogue of the Flora of the Canadian Roclin. Mountains and the Selkirk Range.

Frederieq (L.) et Massart (J.), Notice sur Leo Errera.

Gagnepain, Essai d'une classification des Capparis d'Asie.

- Un arbre oleifere d'Indo-Chine. L'huile de Chaulmoogra et le faux Chaulmoogra.

Gillot (X.), Excursion à Beaume-les-Messieurs (Jura).

- Déformation coralloide du Polyporus umbellatus Fr.

T. LVI.

(SÉ.INCES) 21 
Gulia (G.), Intorno ad un nuovo habitat della Melitella pusilla Somm. Hébert (A.) et Heim (F.), Composition et emploi de la pulpe de défribrage de Hennequin.

Heim (F.), Notes de botanique pure et appliquée.

Hitchcock (A.-S.), Catalogue of the grasses of Cuba.

Holm (Theo.), Remarks on the structure and affinities of Dewey's Carices.

- The Gramineæ of the alpine region of the Rocky Mountains in Cỏlorado.

Huber (J.), Materiaes para a Flora amazonica, VII, Plantæ Duckeanæ austro-guyanenses.

Krautter (L.), A comparative Study of the Genus Pentstemon.

Labergerie, Adaptations culturales et variations des Solanées tubérifères.

Le Dantec (F.), La crise du transformisme.

Lemoine (M $\left.\mathbf{M}^{\mathrm{me}} \mathrm{Paul}\right)$, Sur la distinction anatomique des genres Lithothamnium et Lithophyllum.

Marshall Ward (H.), Trees, Vol. V, Form and Habit.

Nakai, Flora Koreana (Pars prima).

Pittier, New or noteworthy plants from Columbia and Central America.

Pyat (F.), L'exposition de Champignons du Jardin des Plantes d'Angers en 1907.

Trelease, The Mexican Fiber Agaves known as Zapupe.

Van Alderwerelt van Rosenburgh, Malayan Ferns.

Vilmorin-Andrieux, Supplément aux Meilleurs Blés.

Der Botanische Garten und das Botanische Museum der Universität Zurich in Jahre 1908.

Recueil d'œuvres de L. Errera. - Botanique générale (2 vol.).

- Mélanges.

Schedx ad herbarium floræ rossicx a Museo Botanico Academix Imperialis Scientiarum Petropolitanæ editæ, VI (n ${ }^{\text {os }}$ 1601-2000).

Tweede Overgicht der Schadelijk an Nattige Insecten von Java.

Mémoires de la Société linnéenne de Normandie, $\mathrm{XIII}^{\mathrm{e}}$ vol., $\mathbf{1}^{\mathrm{er}}$ fasc. 1908.

Mémoires de la Société d'Émulation du Doubs, $8^{\mathrm{e}}$ série, $2^{\mathrm{e}}$ vol., 1907.

Mémoires de l'Académie royale de Belgique, $2^{\mathrm{e}}$ série, こั volumes.

Bulletin de l'Académie royale de Belgique, Sciences, $1908, \mathrm{n}^{\text {os }} 3-12$, 1909, $\mathrm{n}^{\text {os }}$ 1-2.

Recueil des Travaux Botaniques Néerlandais, publiés par la Société Botanique Néerlandaise, V, 2-4. 
heE. - le mastoidea tessellata hook. Fil. et harv.

Mitteilungen an den Botanischen Museum der Universität Zurich, XL et LXIV.

Annali della $\boldsymbol{R}$. Academia d'Agricoltura di Torino, LI, 1908.

Mémoires de l'Académie impériale des Sciences de Saint-Pétersbourg, VIII ${ }^{\mathrm{e}}$ série, XXII, 2, 1907.

Bulletin de l'Académie impériale des Sciences de Saint-Pétersbourg, Ve série, XXV, 1906.

Nïvenytani Közlemények, VII, כ, 1908.

Missouri botanical Garden. - Nineteenth annual Report.

Contribution from the United States National Herbarium (Smithsonian Institution), XII, 7-9.

Bulletin of the scientific Laboratories of Denison University.

Bulletin de l'Agriculture aux Indes Néerlandaises, XXII.

M. F. Camus donne lecture de la Note ci-dessous :

\section{Le Mastoidea tessellata Hook. fil. et Harv.;}

PAR M. L'ABBÉ HUE.

\section{Genus Mastoidea}

Hook. fil. et Harv., apud J. Dalton Hooker Cryptog. Botan. of the antarct. voyage of $H$. M. discovery ships Erebus and Terror, 18391843, unter the comm. of capt. J. Clark Ross, 1840̈, p. 199, Wain. Lich., 1903, p. 36, in Résultats du voyage du a S. Y. Belgica ॰, 18971899, sous le command. de A. de Guerlache de Gommery, Botan., ac Zahlbruckn. Ascolich., 1907, p. 247, apud Engler und Prantl Natürl. Pflanzenfamil.; Hariot Note sur le genre Mastoidea, apud Morot Journ. Botan., t. I, 1887, p. 231 ; Leptogiopsis Nyl. Lich. nov. freti Behring., in Flora, 1884, p. 211, et Enum. Lich . freti Behring., 1888, p. 26, in Bull. Soc. Linn. Normand., $4^{\mathrm{e}}$ sér., t. I, et apud Hue Lich. exot., n. 136, in Nouv. Arch. Mus., $3^{\text {e }}$ sér., t. II, 1890 (non Leptogiopsis Vüll. Arg., Lich. Beitr., n. 372, in Flora 1892); Dermatomeris, Gen. nov. Ulvearum, Reinsch, Süzwasseralgenflor. Süd-Georgien, XIV, p. 35̈8, tab. IV, fig. 12-14; idem, Gen. nov. Mastodiacearum (Lichen.) Reinsch, Meeresalgenflor. Süd-Georgien, XV, p. 24ä, ambo in Internationale Polarforschung, Berlin, 1890.

Thallus erectus, ecorticatus et biformis; primum foliaceus, membranaceus, stipite brevi aut brevissimo stipitatus ac intus homœomericus; dein fruticuloso foliaceus, intus symmetricus, stipitatus stipite tereti et plus minusve longo et superne foliosus foliis mox aggregatis atque tunc capitatus capite extus granuloso sorediato (fig. 1). Gonidia chlorophycea. 


\section{$2 \mathrm{BHL}$ Biodiversity Heritage Library}

1909. "Séance Du 11 Juin 1909." Bulletin de la Société botanique de France 56, 313-315. https://doi.org/10.1080/00378941.1909.10831414.

View This Item Online: https://www.biodiversitylibrary.org/item/8678

DOI: https://doi.org/10.1080/00378941.1909.10831414

Permalink: https://www.biodiversitylibrary.org/partpdf/160802

\section{Holding Institution}

Missouri Botanical Garden, Peter H. Raven Library

\section{Sponsored by}

Missouri Botanical Garden

\section{Copyright \& Reuse}

Copyright Status: Public domain. The BHL considers that this work is no longer under copyright protection.

This document was created from content at the Biodiversity Heritage Library, the world's largest open access digital library for biodiversity literature and archives. Visit BHL at https://www.biodiversitylibrary.org. 